\title{
The value of LH supplementation in young women with diminished ovarian reserve treated with GnRH Antagonist Protocol for ovarian hyperstimulation in ICSI-cycles
}

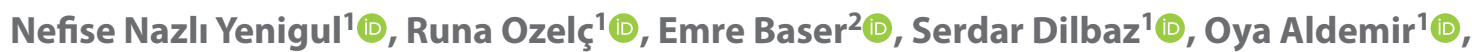 \\ Berna Dilbaz $^{1}$, Ozlem Moraloğlu Tekin ${ }^{1}(\mathbb{D}$ \\ ${ }^{1}$ IVF Clinic of the University of Health Sciences School of Medicine, Etlik Zubeyde Hanım Research and Training Hospital, \\ Ankara, Turkey \\ ${ }^{2}$ Yozgat Bozok University Faculty of Medicine, Department of Obstetrics and Gynecology, Yozgat, Turkey
}

\begin{abstract}
Objectives: To compare the success of two controlled ovarian hyperstimulation protocols; rFSH + hp-hMG with only rFSH in the $\mathrm{GnRH}$ antagonist protocol in diminished ovarian reserve under 35 years of age.

Material and methods: Data from January 2015 to June 2019 were abstracted from the hospital records of IVF Clinic. The women younger than 35 years of age who were diagnosed as diminished ovarian reserve and underwent standard GnRH antagonist protocol were included. Patients in Group-1 underwent controlled ovarian stimulation with rFSH alone and Group-2 with rFSH in combination with hp-hMG. Patients in both groups were divided into three subgroups according to their antral follicle count at Day 3: < 4 (a), 4-6 (b), and 7-10 (c). Demographic features and IVF outcomes of the patients were extracted.
\end{abstract}

Results: Total number of retrieved oocytes, was higher in Group-1 than Group-2 (6.5 \pm 2.1 vs $5.5 \pm 2.3$, respectively, p $<0.001)$. However, there were no significant differences between the two groups in terms of clinical pregnancy rate, implantation rate, miscarriage rate and live birth rate. Although the main study outcome parameters did not show significant difference between Group-1a and Group-2a, the number of mature oocytes (5 \pm 2.8 vs $1.8 \pm 1.2$, respectively, $p=0.006)$ was higher in Group-1a.

Conclusions: We observed no beneficial effect of LH supplementation during IVF for the treatment of women under 35 years old with diminished ovarian reserve in the first treatment cycle when compared with rFSH only in the antagonist protocols. Key words: diminished ovarian reserve; GnRH antagonist; luteinizing hormone; ovarian hyperstimulation

\section{INTRODUCTION}

An adequate ovarian response to controlled ovarian stimulation (COS) is crucial for the success of assisted reproductive technology (ART). Follicle-stimulating hormone (FSH) and luteinizing hormone (LH) are known to play pivotal roles in folliculogenesis. Although LH is required in the follicular phase to ensure both follicular estradiol (E2) production necessary for completion of oocyte maturation and endometrial preparation for implantation, treatment with FSH alone generally leads to successful development and maturation of follicles during in vitro fertilization (IVF) cycles [1-3]. Some recent studies suggested that LH supplementation at the mid-follicular phase of controlled ovarian stimulation during IVF treatment might improve pregnancy rates, however this topic is still debatable, as the data from different studies is conflicting. Gonadotropin agonists or antagonists are used to suppress the pituitary secretion of gonadotropins and to have a complete control of the follicular phase of the treatment cycle with administration of exogenous gonadotropins. These agonist and antagonist 
regimens aim to prevent early luteinization of the follicle by suppression of an early LH rise. During the last decade, the use of $\mathrm{GnRH}$ antagonist protocols provides the opportunity to control the endogenous LH surge in a rapid and more convenient way and thereby cause immediate suppression of gonadotrophin release without the so-called flare-up effect. Previous studies investigated the role of LH supplementation in GnRH agonists protocols in IVF-ET treatment cycles [3-5]. However, the efficacy of LH support in $\mathrm{GnRH}$ antagonist protocols is still unclear.

Although FSH alone can induce follicular growth, based on the 'two cell two gonadotropin' theory'; presence of LH in the follicular phase of a natural cycle is important for production of estradiol. Estradiol is secreted by FSH stimulation in granulosa cells from the androgenic precursors secreted from the theca cells in response to $\mathrm{LH}$ stimulation. Moreover, $\mathrm{LH}$ has a role in the resumption of meiosis and in production of progesterone following ovulation in order to sustain the integrity of the endometrium. In normogonadotropic women, although the pituitary gland $\mathrm{LH}$ and FSH secretion is down-regulated by gonadotropin agonist or antagonist administration, r-FSH alone leads to acceptable ovarian stimulation as residual $\mathrm{LH}$ is adequate for its pivot role in follicular growth and stimulation. Therefore, stimulation with rec-FSH alone or HMG has similar implantation and pregnancy rates [6].

There are also conflicting opinions regarding the specific patient group that can benefit from LH supplementation in ART cycles [2]. In recent years, there has been a significant increase in the number of young patients with diminished ovarian reserve (DOR) who apply for an IVF-ET treatment.

\section{Objectives}

The effect of LH supplementation during controlled ovarian hyperstimulation in IVF/intracytoplasmic sperm injection (ICSI) cycles in women over 35 years of age who have DOR have been studied [1,7]. However, there have been limited studies on the use of LH in IVF/ICSI cycles in the treatment of women with DOR under 35 years of age. Therefore, in this study, we compared the success of two controlled ovarian hyperstimulation protocols; recombinant follicle-stimulating hormone $(\mathrm{rFSH})+$ highly purified human menotropin (hp-hMG) with only rFSH in the $\mathrm{GnRH}$ antagonist protocol in poor responders under 35 years of age.

\section{MATERIAL AND METHODS}

This study was conducted as a retrospective trial in the IVF Clinic of the University of Health Sciences School of Medicine, Etlik Zubeyde Hanım Research and Training Hospital, Ankara, Turkey, between January 2015 and June 2019. The study protocol was approved by the hospital's Ethics Com- mittee (N: 90057706-799). Informed consent was obtained from all participants.

The women younger than 35 years of age who were diagnosed as DOR and underwent standard GnRH antagonist protocol were included in the study. DOR was defined as having one of the criteria defined below: antral follicle count $(\mathrm{AFC})<11$, Antimüllerian Hormone $(\mathrm{AMH})<1.1 \mathrm{ng} / \mathrm{mL}$ and number of collected oocytes $<7[4,7]$. The exclusion criteria were being a normo-responder, high responders or $\geq 35$ years old poor responder or having a body mass index $(\mathrm{BMI}) \geq 30$ or organic lesion of the endometrium or uterus, patients with ovarian cyst or ovarian surgery, patients with grade 3-4 endometriosis, uterine abnormality, or a history of radiotherapy and chemotherapy.

All patients were scheduled for their first ICSI-embryo transfer (ET) cycles. Patients in Group-1 underwent controlled ovarian stimulation with rFSH (Gonal-F; MerckSerono, SA, Geneva, Switzerland) alone and Group-2 with rFSH (Gonal-F; MerckSerono, SA, Geneva, Switzerland) in combination with hp-hMG (Merional, IBSA, Switzerland). Due to the different clinical approaches of clinicians in our clinic, hp-HMG was added to some DOR patients under the age of 35 starting from the first day of their treatment. Patients in both groups were divided into three subgroups according to their AFC at Day 3: < 4 (a), 4-6 (b), and 7-10 (c).

Data of the patient groups; age, body mass index (BMI), total dose of rFSH and rLH administered, duration of induction, serum E2 levels and progesterone at hCG administration, number of follicles $<11 \mathrm{~mm}$, number of total follicles and number of follicles measuring $>16 \mathrm{~mm}$ at hCG administration, endometrial thickness at retrieval, total number of oocytes retrieved, numbers of mature oocytes (MII); number of oocytes fertilized, number of embryos obtained were extracted from the electronic patient files.

For all participants, baseline ultrasound scan and blood tests were performed on day 2-3 of the menstrual cycle. Ovarian stimulation with gonadotropins was commenced with a dose of 225-400 IU, and subjects were monitored every $2-4$ days by transvaginal ultrasound scan (TVUS) (Logiq A5; General Electric, Milwaukee, WI) performed by the same physician starting on day five of ovarian hyperstimulation. GnRH antagonist (0.25 mg/Day of Cetrotide; Merck-Serono, Darmstadt, Germany) was initiated when the leading follicle reached 13-14 mm on TVUS or when the serum E2 level was $>400 \mathrm{ng} / \mathrm{mL}$. Cycle cancelation was defined as lack of follicular development (no follicles of at least $12 \mathrm{~mm}$ after eight days of treatment and endometrial thickness $<4 \mathrm{~mm}$ ) and/or a clinically significant decrease in serum E2 level in two consecutive days during the ovarian stimulation period. When at least three follicles reached a mean diameter of $\geq 18 \mathrm{~mm}, 250 \mu \mathrm{g}$ of recombinant human chorionic gonadotropin (rHCG) (Ovitrelle; Merck Serono, Germany) 
or 10000 IU of urinary HCG (Pregnyl; Schering-Plough, Turkey) was administered. Transvaginal ultrasound-guided oocyte retrieval was performed 35.5 hours after HCG administration. Oocyte pick-up was performed under general anesthesia. The oocytes were inseminated by ICSI. The luteal phase was supported with progesterone vaginal gel (90 mg/d, Crinone 8\% Vaginal Gel; Merck-Serono, Vevey, Switzerland) twice a day after oocyte pick-up and continued in pregnant patients until 12 weeks of gestation.

The primary endpoints of the study were implantation rate (IR; positive $\beta$-hCG test $\geq 10 \mathrm{IU}, 10$ days after embryo transfer), clinical pregnancy rate (CPR) (determination of embryonic heartbeat on TVS was defined as clinical pregnancy), miscarriage rate (MR; pregnancy loss before 24 weeks of gestation) and live birth rate (LBR; delivery of a viable infant after 24 weeks of gestation). Secondary outcomes were the total number of retrieved oocytes, the number of mature oocytes and number of good-quality embryos.

\section{Statistics}

Statistical analyses were performed using SPSS (version 20; SPSS, Chicago, IL). Data are expressed as the average \pm SD or percentage. The variables were investigated using visual (histograms, probability plots) and analytical methods (Kolmogorov-Smirnov/Shapiro-Wilk test) to determine whether they were normally distributed. The Mann-Whitney U-test was used for non-parametric numerical data, and Student's $t$-test was used for parametric data. Categorical data were compared using the Chi-square test or Fisher's exact test. In all analyses, $P<0.05$ was taken to indicate statistical significance.

\section{RESULTS}

During the study period a total of 393 women under age 35 who were diagnosed as DOR were treated at our institution; 119 consecutive patients treated with rFSH (Group-1) and 274 consecutive patients receiving rFSH $+\mathrm{hp}-\mathrm{hMG}$ treatment (Group-2) were included in the study. Patients' demographic characteristics and ovarian stimulation cycle outcomes are shown in Table 1. Demographic parameters (age, BMI, and parity) were similar in the two groups. Duration of ovarian stimulation ( $9.4 \pm 2$ vs $8.6 \pm 1.9$ days, respectively, $\mathrm{p}<0.001)$, total dose of gonadotropins administered $(2738.3 \pm 989.4$ vs $1913.1 \pm 575.1 \mathrm{IU}$, respectively, $\mathrm{p}<0.001)$ were higher in Group-2 in comparison to Group-1. In contrast, basal LH ( $5.5 \pm 3.2$ vs $5.1 \pm 3.5$, respectively, $\mathrm{p}<0.001)$, and E2 level on hCG day (1620.1 \pm 1006.7 vs $1279.9 \pm 673$, respectively, $p=0.001$ ) were higher in group-1 in comparison to Group-2. Basal FSH (10.1 \pm 7 vs $10.7 \pm 3.1$, respectively, $p=0.980)$, Basal E2 (47. \pm 26.5 vs $48.7 \pm 49.6$, respectively, p: 0.267$), \operatorname{AFC}(7.0 \pm 2.7$ vs $7.5 \pm 2.1$, respectively, p: 0.647$)$,
$\mathrm{AMH}(0,4 \pm 0.1$ vs $0.3 \pm 0.2$, respectively, p: 0.645$)$ levels were similar in the two groups (Tab. 1).

The main study outcome total number of retrieved oocytes, was higher in Group-1 (rFSH group) than Group-2 ( $6.5 \pm 2.1$ vs $5.5 \pm 2.3$, respectively, $\mathrm{p}<0.001)$ (Tab. 1, Fig. 1). There were no significant differences between the two groups in terms of CPR, IR, MR, LBR and multiple pregnancy rate (twins) (Tab. 1).

The main data set was analyzed according to different ranges of AFC, i.e., < 4 (subgroup a), 4-6 (subgroup b), and 7-10 (subgroup c) (Tab. 2). The majority of both laboratory clinical and clinical outcome measures were comparable in subgroup c (AFC 7-10), regardless of the stimulation regimen (Group-1c and Group-2c), but in this subgroup the percentage of the total number of retrieved oocytes was moderately higher in Group-1c than Group-2c (6.5 \pm 2.2 vs $6.3 \pm 1.9$, respectively, p:0.014). In addition, total gonadotropin doses in Group-2c (2775.2 \pm 953.4 vs $2040.4 \pm 548.7$, respectively, $p<0.001)$ were higher, and E2 values on HCG Day $(1447.7 \pm 564.6$ vs $1794.5 \pm 1075.1$, respectively, $\mathrm{p}=0.003$ ) were lower than Group-1c.

In women with AFC within the range of 4-6 (subgroup $b)$, the total number of retrieved oocytes $(6.5 \pm 1.7$ vs $4.4 \pm 2.3$, respectively, $p=0.049$ ) and the number of mature oocytes ( $4.5 \pm 2.6$ vs $3.2 \pm 1.9$, respectively, $p=0.044$ ) were also higher in Group-1b in comparison to Group-2b. In addition, the patients in Group-2 had similar AMH, however significantly higher duration of treatment $(9.3 \pm 2$ vs $9 \pm 1$, respectively, $\mathrm{p}<0.001)$ and total gonadotropin dose $(2826.4 \pm 928.1$ vs $2415 \pm 984.2$, respectively, $p<0.001$ ) in Group-2b. There were no marked differences between Group-1a and Group-2a (women with AFC < 4). Although the main study outcome parameters did not show significant difference between Group-1a and Group-2a, the number of mature oocytes ( $5 \pm 2.8$ vs $1.8 \pm 1.2$, respectively, p: 0.006) was higher in Group-1a.

\section{DISCUSSION}

GnRH antagonist regimens have been widely used in recent years as these regimens are flexible and have a shorter duration of gonadotropin administration. GnRH antagonists down-regulate LH secretion rapidly, but in a reversible manner. The promoting effect of premature high LH levels on follicular atresia is more established yet, the treshold LH level that will support follicular growth without promoting follicular atresia is not quite clear [8, 9]. LH supplementation in patients with suboptimal response have been proposed in order to improve IVF-ET outcome. Although many different protocols and strategies have been proposed for enhancement of the outcome in these patients, there is no consensus on the ideal treatment regimen [10]. 
Table 1. Demographic characteristics and ovarian stimulation outcomes

\begin{tabular}{|c|c|c|c|}
\hline & recFSH & recFSH+HMG & \\
\hline & $119(30.3 \%)$ & $274(69.7 \%)$ & $\mathbf{p}$ \\
\hline Age [years] & $29.3 \pm 3.1$ & $29.6 \pm 3.3$ & 0.316 \\
\hline BMI $\left[\mathrm{kg} / \mathrm{m}^{2}\right]$ & $27.7 \pm 5.2$ & $27.1 \pm 5.2$ & 0.352 \\
\hline Basal FSH [IU/L] & $10.7 \pm 3.1$ & $10.1 \pm 7$ & 0.980 \\
\hline Basal LH [IU/L] & $5.5 \pm 3.2$ & $5.1 \pm 3.5$ & 0.047 \\
\hline Basal E2 [pmol/L] & 47. \pm 26.5 & $48.7 \pm 49.6$ & 0.267 \\
\hline AFC & $7.0 \pm 2.7$ & $7.5 \pm 2.1$ & 0.647 \\
\hline AMH & $0,4 \pm 0.1$ & $0.3 \pm 0.2$ & 0.645 \\
\hline Days of stimulation & $8.6 \pm 1.9$ & $9.4 \pm 2$ & $<0.001$ \\
\hline Total gonadotropin dose [IU] & $1913.1 \pm 575.1$ & $2738.3 \pm 989.4$ & $<0.001$ \\
\hline E2 value on HCG day [pmol/L] & $1620.1 \pm 1006.7$ & $1279.9 \pm 673$ & 0.001 \\
\hline $\mathrm{P}$ value on $\mathrm{HCG}$ day $[\mathrm{pmol} / \mathrm{L}]$ & $0.9 \pm 0.4$ & $1.2 \pm 2.9$ & 0.252 \\
\hline Endometrial thickness $[\mathrm{mm}]$ on HCG day & $9.7 \pm 2$ & $9.9 \pm 2.1$ & 0.518 \\
\hline Number of follicles $\geq 17 \mathrm{~mm}$ in diameter on HCG day & $2.8 \pm 2$ & $2.1 \pm 1.5$ & 0.005 \\
\hline Number of follicles $15-17 \mathrm{~mm}$ in diameter on HCG day & $2.6 \pm 2.5$ & $1.9 \pm 1.6$ & 0.038 \\
\hline LH value on OPU day [pmol/L] & $3.2 \pm 4.3$ & $3.4 \pm 4.0$ & 0.996 \\
\hline No. of retrieved oocytes & $6.5 \pm 2.1$ & $5.5 \pm 2.3$ & $<0.001$ \\
\hline No. of mature oocytes & $4.9 \pm 2.2$ & $4.1 \pm 2.2$ & 0.005 \\
\hline Fertilization rate & $0.5 \pm 0.3$ & $0.5 \pm 0.3$ & 0.764 \\
\hline No. of $2 \mathrm{PN}$ & $2.8 \pm 1.7$ & $2.3 \pm 1.8$ & 0.012 \\
\hline No of good quality embryo & $1.1 \pm 0.7$ & $0.90 \pm 0.6$ & 0.043 \\
\hline Transferred embryos (mean \pm SD) & $0.9 \pm 0.8$ & $0.7 \pm 0.7$ & 0.018 \\
\hline No. of cancelled cycles & $13(11.5 \%)$ & $26(10.8 \%)$ & 0.841 \\
\hline Implantation & $27(46.6 \%)$ & $58(41.1 \%)$ & 0.483 \\
\hline Clinical pregnancy & $24(41.4 \%)$ & $48(34.0 \%)$ & 0.328 \\
\hline Live birth & $17(29.3 \%)$ & $33(23.4 \%)$ & 0.383 \\
\hline Misscarriage & $5(21.7 \%)$ & $8(19.0 \%)$ & 0.795 \\
\hline Multiple pregnancy & $0(0.0 \%)$ & $4(9.5 \%)$ & 0.127 \\
\hline
\end{tabular}

Values were presented as mean \pm SD. P value $<0.05$ was statistically significant; SD — standard deviation; BMI — body mass index; AFC — antral follicle count; AMH — antimullerian hormone; $\mathrm{FSH}$ - follicle stimulating hormone; LH — luteinizing hormone; E2 — estradiol; P — progesterone; HCG — human chorionic gonadotropin; OPU — oocyte pick-up; ET — embryo transfer; PN — Pronucleus

Younger women with low ovarian reserve represent one of the most difficult groups to treat, so the administration of ideal gonadotropin protocol is especially important for this group. Several attempts have been made to improve clinical outcomes, such as increasing the initial dose of rFSH or using different GnRH analogs or supplementation of standard FSH treatment with LH/hMG [11]. Despite some overlap among studies, conflicting results have been reported. Alviggi et al., [12] reported that LH supplementation might be beneficial in patients with adequate pre-stimulation ovarian reserve parameters that show an unexpected low response to FSH monotherapy and women with an age range of 36 to 39 . Alviggi et al. [12], reported no significant success with addition of LH in DORs patients defined according to the Bologna criteria. In a meta-analysis [8], a significantly higher clinical pregnancy (odds ratio: $2.03, \mathrm{p}=0.003$ ), implantation rate (odds ratio: $2.62, \mathrm{p}=0.004$ ) and number of oocytes retrieved (weight mean difference: $1.98, \mathrm{p}=0.03$ ) was achieved in hypo-responders supplemented with recombinant $\mathrm{LH}(\mathrm{rLH})$ when compared with DOR patients undergoing FSH monotherapy. However, the heterogeneity of the patients included in the studies reviewed makes it difficult to determinate the characteristic of women who would benefit from LH supplementation. The presented study demonstrated that addition of hp-hMG to rFSH on the day of gonadotropin administration had no significant effect on implantation rate, clinical pregnancy rate or live birth rates using the flexible $\mathrm{GnRH}$ antagonist protocol in women with DOR aged under 35 years. In addition, the total gonadotropin dose was lower in patients receiving only $\mathrm{rFSH}$ in all subgroups. 


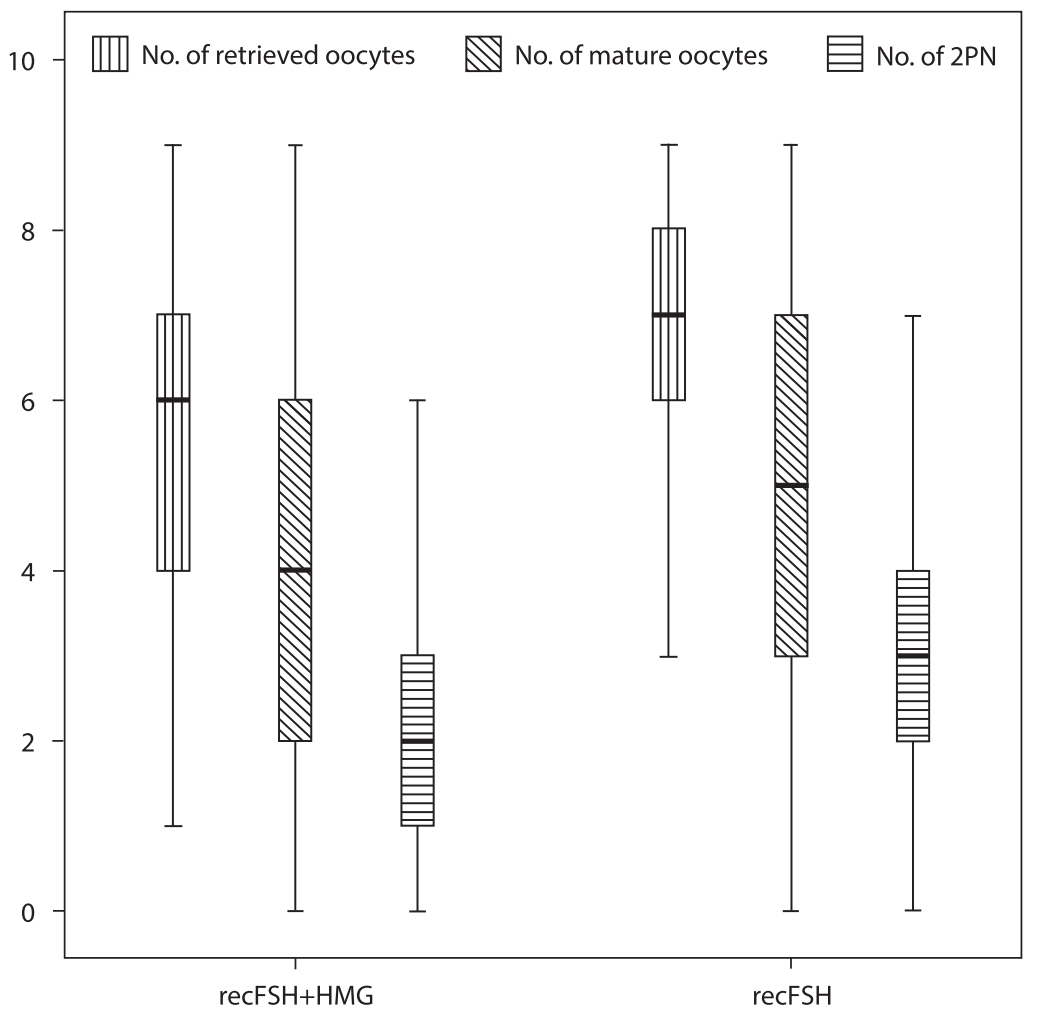

Figure 1. Ovarian stimulation outcomes and comparison between the recFSH+HMG or recFSH treatment

Although most recent publications have shown that LH supplementation to FSH makes measurable differences in clinical practice $[8,11,12]$, the study by Vuong et al., [1] showed different results as LH supplementation to FSH using a GnRH antagonist protocol had no significant effect on the total number of retrieved oocytes, mature oocytes, implantation rate, clinical pregnancy rate or live birth rate in women aged $\geq 35$ years. In contrast, in our study, total numbers of retrieved and mature oocytes were even higher in the rFSH group in women $<35$ years with DOR. However, although the number of good-quality embryos was better in the rFSH group, implantation rates were similar. The difference results obtained by Vuong et al. [1], might be related to the recruitment of patients with better prognosis as women with AFC $>10$ were also included in the study group, and the mean AMH was 1.6.

In a similar study in which patients under the age of 35 were also evaluated [13], retrieved and mature oocyte counts were similar in patients who received rFSH only and rFSH + hp-hMG, but implantation and clinical pregnancy rates were higher in the $\mathrm{rFSH}+\mathrm{hp}-\mathrm{hMG}$ group. The participants included in this study had an age range of 19 to
42 and unlike the presented study the study group was not restricted to a hypo-responder group.

Similar to the Voung's study [1], the number of cancelled cycles was lower in patients with LH supplementation in our study group. Our finding of significantly higher levels of estradiol in patients receiving rec-FSH only, supports the hypothesis that states that endogenous serum LH level at the late-follicular phase is sufficient even if a GnRH antagonist have been started. Younnis et al. [14], supplemented LH on the day of hCG trigger but failed to demonstrate any difference in E2 levels when compared to the levels without LH supplementation in antagonist cycles in women $<35$ years of age and/or had a previous low ovarian response. According to the findings of the presented study, it can be speculated that the total number of retrieved oocytes and mature oocyte counts of the patients in the rFSH group was high due to the higher E2 levels. Contrarily, progesterone $(P)$ levels on hCG day were similar in two groups, in concordance with other published studies $[13,15]$. Bosch et al., [16] found a high P level on hCG administration day in all patients without LH supplementation and reported a significant increase in implantation rate after LH supplementation 
Table 2. Comparison between the recFSH+HMG or recFSH treatment subpopulations of patients characterised by different ranges of AFC, $<4$, 4-6 and 7-10

\begin{tabular}{|c|c|c|c|c|c|c|c|c|c|}
\hline & \multicolumn{3}{|c|}{$<4$ AFC (n: 53) } & \multicolumn{3}{|c|}{ 4-6 AFC (n: 85) } & \multicolumn{3}{|c|}{$>6$ AFC (n: 253) } \\
\hline & $\begin{array}{l}\text { recFSH } \\
(n: 9)\end{array}$ & $\begin{array}{l}\text { recFSH } \\
+ \text { HMG } \\
\text { (n:44) }\end{array}$ & $\mathbf{P}$ & $\begin{array}{l}\text { recFSH } \\
(\mathrm{n}: 12)\end{array}$ & $\begin{array}{l}\text { recFSH } \\
+ \text { HMG } \\
(n: 73)\end{array}$ & $\mathbf{p}$ & $\begin{array}{l}\text { recFSH } \\
(\mathrm{n}: 96)\end{array}$ & $\begin{array}{l}\text { recFSH } \\
+ \text { HMG } \\
\text { (n: 157) }\end{array}$ & $\mathbf{p}$ \\
\hline Age [years] & $32.4 \pm 1.1$ & $32.2 \pm 1.2$ & 0.234 & $32.8 \pm 0.8$ & $32.8 \pm 1.1$ & 0.419 & $32.4 \pm 1$ & $32.9 \pm 1.1$ & 0.131 \\
\hline BMI $\left[\mathrm{kg} / \mathrm{m}^{2}\right]$ & $25.4 \pm 4.3$ & $26.2 \pm 4.1$ & 0.486 & $26.1 \pm 7.1$ & $26.6 \pm 4.9$ & 0.763 & $28.3 \pm 5.2$ & $26.3 \pm 5.5$ & 0.139 \\
\hline AFC & $2.1 \pm 0.9$ & $2.6 \pm 0.8$ & 0.149 & $4.7 \pm 0.7$ & $5.4 \pm 0.7$ & 0.053 & $8.8 \pm 0.9$ & $9.1 \pm 1.0$ & 0.101 \\
\hline $\mathrm{AMH}$ & $0.3 \pm 0.2$ & $0.4 \pm 0.1$ & 0.913 & $0.5 \pm 0.2$ & $0.5 \pm 0.3$ & 0.830 & $0.9 \pm 0.1$ & $0.9 \pm 0.2$ & 0.532 \\
\hline Days of stimulation & $6.6 \pm 2.9$ & $7.9 \pm 2.4$ & 0.903 & $9 \pm 1$ & $9.3 \pm 2$ & $<0.001$ & $9.1 \pm 1.7$ & $9.9 \pm 1.9$ & 0.154 \\
\hline Total gonadotropin dose [IU] & $\begin{array}{c}1995 \\
\pm 897.8\end{array}$ & $\begin{array}{r}3078.6 \\
\pm 1115.3\end{array}$ & 0.012 & $\begin{array}{c}2415 \\
\pm 984.2\end{array}$ & $\begin{array}{r}2826.4 \\
\pm 928.1\end{array}$ & $<0.001$ & $\begin{array}{r}2040.4 \\
\pm 548.7\end{array}$ & $\begin{array}{l}2775.2 \\
\pm 953.4\end{array}$ & $<0.001$ \\
\hline E2 value on $\mathrm{HCG}$ day [pmol/L] & $\begin{array}{c}626.3 \\
\pm 525.7\end{array}$ & $\begin{array}{c}420.3 \\
\pm 236.2\end{array}$ & 0.230 & $\begin{array}{r}1494.5 \\
\pm 206.3\end{array}$ & $\begin{array}{r}1078.8 \\
\pm 490.5\end{array}$ & 0.185 & $\begin{array}{c}1794.5 \\
\pm 1075.1\end{array}$ & $\begin{array}{r}1447.7 \\
\pm 564.6\end{array}$ & 0.003 \\
\hline$P$ value on $\mathrm{HCG}$ day $[\mathrm{pmol} / \mathrm{L}]$ & $0.8 \pm 0.2$ & $0.7 \pm 0.5$ & 0.909 & $1 \pm 0.8$ & $0.8 \pm 0.5$ & 0.476 & $1 \pm 0.5$ & $2.2 \pm 6$ & 0.300 \\
\hline $\begin{array}{l}\text { Endometrial thickness [mm] } \\
\text { on HCG day }\end{array}$ & $9.2 \pm 1.1$ & $9.5 \pm 1.6$ & 0.517 & $10.1 \pm 2.9$ & $9.5 \pm 2.1$ & 0.331 & $10 \pm 2$ & $10.1 \pm 2.1$ & 0.681 \\
\hline $\begin{array}{l}\text { Number of follicles } \geq 17 \mathrm{~mm} \\
\text { in diameter on HCG day }\end{array}$ & $1 \pm 0.8$ & $1.5 \pm 1.2$ & 0.398 & $2.2 \pm 1.3$ & $2 \pm 1.5$ & 0.414 & $3 \pm 2$ & $2.3 \pm 1.5$ & 0.035 \\
\hline $\begin{array}{l}\text { Number of follicles } 15-17 \mathrm{~mm} \\
\text { in diameter on HCG day }\end{array}$ & $0.7 \pm 0.8$ & $1.2 \pm 1.3$ & 0.440 & $2.1 \pm 1.7$ & $1.8 \pm 1.8$ & 0.434 & $2.8 \pm 2.6$ & $2.2 \pm 1.5$ & 0.182 \\
\hline No. of retrieved oocytes & $5.5 \pm 3.5$ & $2.9 \pm 2.7$ & 0.132 & $6.5 \pm 1.7$ & $4.4 \pm 2.3$ & 0.049 & $6.5 \pm 2.2$ & $6.3 \pm 1.9$ & 0.014 \\
\hline No. of mature oocytes & $5 \pm 2.8$ & $1.8 \pm 1.2$ & 0.006 & $4.5 \pm 2.6$ & $3.2 \pm 1.9$ & 0.044 & $4.3 \pm 2.5$ & $5 \pm 2$ & 0.507 \\
\hline Fertilization rate [\%] & $0.7 \pm 0$ & $0.6 \pm 0.5$ & 0.432 & $0.6 \pm 0.2$ & $0.6 \pm 0.4$ & 0.315 & $0.5 \pm 0.3$ & $0.5 \pm 0.3$ & 0.356 \\
\hline No. of $2 \mathrm{PN}$ & $3.5 \pm 2.1$ & $1 \pm 1.1$ & 0.104 & $3 \pm 0.8$ & $2.2 \pm 1.8$ & 0.066 & $2.4 \pm 1.7$ & $2.8 \pm 2.1$ & 0.713 \\
\hline Implantation & $3(75.0 \%)$ & $5(41.7 \%)$ & 0.248 & $2(22.2 \%)$ & $21(47.7 \%)$ & 0.160 & $21(47.7 \%)$ & $32(37.6 \%)$ & 0.270 \\
\hline Clinical pregnancy & $2(50.0 \%)$ & $5(41.7 \%)$ & 0.771 & $2(22.2 \%)$ & $17(38.6 \%)$ & 0.349 & $12(54.5 \%)$ & $22(35.5 \%)$ & 0.155 \\
\hline Live birth & $1(25.0 \%)$ & $3(25.0 \%)$ & $<0.001$ & $2(22.2 \%)$ & $11(25.0 \%)$ & 0.860 & $14(31.8 \%)$ & $19(22.4 \%)$ & 0.243 \\
\hline Misscarriage & 1 (50.0\%) & $2(40.0 \%)$ & 0.809 & $0(0 \%)$ & $6(35.3 \%)$ & 0.310 & 4 (21.1\%) & $5(20.0 \%)$ & 0.932 \\
\hline
\end{tabular}

Values were presented as mean \pm SD. P value $<0.05$ was statistically significant; SD — standard deviation; BMI — body mass index; AFC — antral follicle count; AMH — antimullerian hormone; $\mathrm{FSH}$ - follicle stimulating hormone; $\mathrm{LH}$ - luteinizing hormone; $\mathrm{E} 2$ - estradiol; $\mathrm{P}$ - progesterone; HCG - human chorionic gonadotropin; $\mathrm{OPU}$ - oocyte pick-up; ET — embryo transfer; PN — Pronucleus

in patients aged between $35-39$ years while there was no impact of LH supplementation in patients $<35$ years of age.

Another controversial issue is determining when to starting LH supplementation and several different treatment approaches have been reported. In some studies, showing a positive effect of the addition of $\mathrm{LH}$ on clinical pregnancy rates in patients over 35 years old [1, 15], rec-LH was administrated after the sixth day considering the activation of LH receptors. In another review [8], LH supplementation started at the mid-follicular phase in hypo-responder patients $>35$ years of age had a positive effect on ART results. Also, younger women (aged 20-36 years) were shown to produce higher quality embryos after addition of $\mathrm{rLH}$ to rFSH treatment on the first day of the $\mathrm{GnRH}$ antagonist stimulation protocol [17]. On the other hand, there are some reports [18] showing that LH supplementation increases the implantation rate independent from the age of the patient. In the current study, LH was added to FSH in the form of
hp-hMG starting from the first day of the treatment, but significantly more high-grade embryos were observed in the $\mathrm{rFSH}$ group. A recent study showed that [19], despite severe limitations, antagonist administration given to selected patients did not have an impact on cycle cancellation rates, the incidence of early luteinization, and overall, ART outcomes. As in many clinics, antagonist administration and LH supplementation are initiated regardless of the serum LH measurements. A better approach might be measuring the $\mathrm{LH}$ values of the patients and only deciding about $\mathrm{LH}$ supplementation if required after finding a threshold value of LH for good IVf outcome.

Unlike other studies, total gonadotropin doses and days of stimulation were lower in patients receiving $\mathrm{rFSH}$ in all subgroups. Especially in the subgroup $a$ and b, although clinical pregnancy rates were similar, total gonadotropin doses were lower in the rFSH group. On the other hand, the greater number of follicles $>17 \mathrm{~mm}$ in the FSH group was 
indicative of a higher number of retrieved oocytes. Therefore, more oocytes were collected with a lower dose of gonadotropin and with a shorter period of stimulation. In this patient group, only rFSH treatment seemed to be more advantageous in terms of cost-effectiveness. The data of patients under 35 years of age in a recent study [16] supported our findings. Hypo-responsiveness to gonadotropin stimulation in the patient group with poor response has been suggested to be linked with genetic mutations or single nucleotide polymorphisms (SNPs) of endogenous gonadotropin receptors and these patients may demonstrate an ovarian sensitivity to exogenous gonadotropins [8].

Among the strengths of the present study was the selected patient group as although the efficacy of adding LH to treatment that appears to be beneficial for the treatment of women with DOR in GnRH agonist protocols has been investigated by many authors $[4,20,21]$, there are few studies with antagonist protocols in women under 35 years old with a diminished ovarian reserve. The similarity of basal E2, AFC and AMH of the two groups increases the validity of the comparison and the interpretation of the data, increasing the strength of our result.

Inclusion of patients receiving their first treatment cycle is the other strength of the study. Moreover, dividing participants into subgroups based on AFCs reduced bias in our definition of DOR. On the other hand, if the number of participants in groups $1 \mathrm{a}$ and $1 \mathrm{~b}$ had been higher, maybe our results might be more significant. The most important limitations of our study are its retrospective design and the use of hMG that has an LH activity derived from hCG rather than LH. Therefore, some authors [12] have not evaluated hMG in their reviews.

\section{CONCLUSIONS}

We observed no beneficial effect of LH supplementation during $\operatorname{COS}$ for the treatment of women under 35 years old with DOR in the first treatment cycle when compared with rFSH only in the antagonist protocols. Randomized control trials focusing specifically on young patients affected by DOR who received antagonist protocols are required for a final decision.

\section{Conflict of interest}

The authors have no conflicts of interest relevant to this article.

\section{REFERENCES}

1. Vuong TNL, Phung HT, Ho MT. Recombinant follicle-stimulating hormone and recombinant luteinizing hormone versus recombinant follicle-stimulating hormone alone during $\mathrm{GnRH}$ antagonist ovarian stimulation in patients aged $\geq 35$ years: a randomized controlled trial. Hum Reprod. 2015; 30(5): 1188-1195, doi: 10.1093/humrep/dev038, indexed in Pubmed: 25740882.
2. Ferraretti AP, La Marca A, Fauser BC, et al. ESHRE working group on Poor Ovarian Response Definition. ESHRE consensus on the definition of,poor response' to ovarian stimulation for in vitro fertilization: the Bologna criteria. Hum Reprod. 2011;26(7): 1616-1624, doi: 10.1093/humrep/der092, indexed in Pubmed: 21505041.

3. Dahan MH, Agdi M, Shehata F, et al. A comparison of outcomes from in vitro fertilization cycles stimulated with either recombinant luteinizing hormone (LH) or human chorionic gonadotropin acting as an LH analogue delivered as human menopausal gonadotropins, in subjects with good or poor ovarian reserve: a retrospective analysis. Eur J Obstet Gynecol Reprod Biol. 2014; 172: 70-73, doi: 10.1016/j.ejogrb.2013.10.027, indexed in Pubmed: 24314801.

4. Mignini Renzini M, Brigante C, Coticchio G, et al. Retrospective analysis of treatments with recombinant FSH and recombinant $\mathrm{LH}$ versus human menopausal gonadotropin in women with reduced ovarian reserve. J Assist Reprod Genet. 2017; 34(12): 1645-1651, doi: 10.1007/s10815017-1034-z, indexed in Pubmed: 28871409.

5. Karlström PO, Holte J, Hadziosmanovic N, et al. Does ovarian stimulation regimen affect IVF outcome? a two-centre, real-world retrospective study using predominantly cleavage-stage, single embryo transfer. Reprod Biomed Online. 2018; 36(1): 59-66, doi: 10.1016/j. rbmo.2017.10.102, indexed in Pubmed: 29233501.

6. Balasch J, Creus M, Fábregues $F$, et al. The effect of exogenous luteinizing hormone (LH) on oocyte viability: evidence from a comparative study using recombinant human follicle-stimulating hormone (FSH) alone or in combination with recombinant LH for ovarian stimulation in pituitary-suppressed women undergoing assisted reproduction. J Assist Reprod Genet. 2001; 18(5): 250-256, doi: 10.1023/a:1016662100572, indexed in Pubmed: 11464575.

7. Nelson SM. Biomarkers of ovarian response: current and future applications. Fertil Steril. 2013; 99(4): 963-969, doi: 10.1016/j.fertnstert.2012.11.051, indexed in Pubmed: 23312225.

8. Conforti A, Esteves SC, Di Rella F, et al. Correction to: The role of recombinant LH in women with hypo-response to controlled ovarian stimulation: a systematic review and meta-analysis. Reprod Biol Endocrinol. 2019; 17(1):31, doi: 10.1186/s12958-019-0475-x, indexed in Pubmed: 30871582.

9. Kumar P, Sait SF. Luteinizing hormone and its dilemma in ovulation induction. J Hum Reprod Sci. 2011; 4(1): 2-7, doi: 10.4103/09741208.82351, indexed in Pubmed: 21772731.

10. Vaiarelli A, Cimadomo D, Ubaldi N, et al. What is new in the management of poor ovarian response in IVF? Curr Opin Obstet Gynecol. 2018; 30(3): 155- 162, doi: $10.1097 / G C O .0000000000000452$, indexed in Pubmed: 29664789.

11. Lehert $\mathrm{P}$, Kolibianakis $E M$, Venetis $C A$, et al. Recombinant human follicle-stimulating hormone ( $\mathrm{r}$-hFSH) plus recombinant luteinizing hormone versus $\mathrm{r}$-hFSH alone for ovarian stimulation during assisted reproductive technology: systematic review and meta-analysis. Reprod Biol Endocrinol. 2014; 12: 17, doi: 10.1186/1477-7827-12-17, indexed in Pubmed: 24555766.

12. Alviggi C, Conforti A, Esteves SC, et al. International Collaborative Group for the Study of r-hLH (iCOS-LH). Recombinant luteinizing hormone supplementation in assisted reproductive technology: a systematic review. Fertil Steril. 2018; 109(4):644-664, doi: 10.1016/j.fertnstert.2018.01.003, indexed in Pubmed: 29653717.

13. Kan O, Simsir C, Atabekoglu CS, et al. The impact of adding hp-hMG in r-FSH started GnRH antagonist cycles on ART outcome. Gynecol Endocrinol. 2019; 35(10): 869-872, doi: 10.1080/09513590.2019.1600667, indexed in Pubmed: 30973022.

14. Younis JS, Izhaki I, Ben-Ami M. The effect of rLH supplementation to the $\mathrm{GnRH}$-antagonist protocol on endocrine dynamics in the advanced reproductive age. J Endocrinol Invest. 2017; 40(8): 831-839, doi: 10.1007/s40618-017-0618-x, indexed in Pubmed: 28290093.

15. König TE, van der Houwen LEE, Overbeek A, et al. Recombinant LH supplementation to a standard $\mathrm{GnRH}$ antagonist protocol in women of 35 years or older undergoing IVF/ICSI: a randomized controlled multicentre study. Hum Reprod. 2013; 28(10): 2804-2812, doi: 10.1093/humrep/det266, indexed in Pubmed: 23838159.

16. Bosch E, Labarta E, Crespo J, et al. Impact of luteinizing hormone administration on gonadotropin-releasing hormone antagonist cycles: an age-adjusted analysis. Fertil Steril. 2011; 95(3): 1031-1036, doi: 10.1016/j.fertnstert.2010.10.021, indexed in Pubmed: 21067717.

17. Wiser A, Hourvitz A, Yinon $Y$, et al. Recombinant human luteinizing hormone supplementation may improve embryo quality in in vitro fertilization/intracytoplasmic sperm injection cycles with gonadotropin-releasing hormone antagonist protocol. Open Journal of Obstetrics and Gynecology. 2011; 1(2): 31-35, doi: 10.4236/ojog.2011.12007. 
18. Borges E, Braga DP, Azevedo Md, et al. Improving implantation rate by adding recombinant $\mathrm{LH}$ supplementation to recombinant FSH during controlled ovarian stimulation in $\mathrm{GnRH}$ antagonist regimen. Fertility and Sterility. 2019; 112(3): e182, doi: 10.1016/j. fertnstert.2019.07.592.

19. Liu M, Liu S, Li L, et al. LH levels may be used as an indicator for the time of antagonist administration in GnRH antagonist protocols-A proof-Of-concept study. Front Endocrinol (Lausanne). 2019; 10: 67, doi: 10.3389/fendo.2019.00067, indexed in Pubmed: 30809195.
20. Marrs R, Meldrum D, Muasher $S$, et al. Randomized trial to compare the effect of recombinant human FSH (follitropin alfa) with or without recombinant human $\mathrm{LH}$ in women undergoing assisted reproduction treatment. Reprod Biomed Online. 2004; 8(2): 175-182, doi: 10.1016/s1472-6483(10)60513-5, indexed in Pubmed: 14989794.

21. Matorras R, Prieto B, Exposito A, et al. Mid-follicular LH supplementation in women aged 35-39 years undergoing ICSI cycles: a randomized controlled study. Reprod Biomed Online. 2009; 19(6): 879-887, doi: 10.1016/j.rbmo.2009.09.016, indexed in Pubmed: 20031032 